EPJ Web of Conferences 53, 02001 (2013)

DOI: $10.1051 /$ epjconf/20135302001

(C) Owned by the authors, published by EDP Sciences, 2013

\title{
UHECR theory and phenomenology: Summary and outlook
}

\author{
Angela V. Olinto ${ }^{\mathrm{a}}$
}

\author{
Department of Astronomy \& Astrophysics, Kavli Institute for Cosmological Physics, Enrico \\ Fermi Institute, The University of Chicago, Chicago, Illinois 60637, USA
}

\begin{abstract}
Theorists and phenomenologists have contributed significantly to the development of the field of ultrahigh energy cosmic rays (UHECRs). Great progress has been achieved in modeling hadronic interactions, developing precise propagation codes, understanding the role of different backgrounds and magnetic fields in the propagation of ultrahigh energy protons and nuclei, predicting the flux of secondary neutrinos and photons, modeling astrophysical sources and their acceleration mechanisms, developing new techniques to test anisotropies in the sky distribution, proposing new physics phenomena that can be tested at ultrahigh energy, and sharpening the distinction between astrophysical interpretations of unexpected trends (such as the composition at the highest energies) and new physics at play in hadronic interactions at energies well beyond the reach of terrestrial laboratories. Better developed models when combined with recent data have framed current open questions. 1. Is the spectral feature at the highest energies the GZK cutoff or the effect of $E_{\max }$ ? 2. Is the composition of primaries changing at the highest energies or are new interactions responsible for the change in behavior of extensive air showers? 3. At what energy and sensitivity will sources be observed? 4. At what energies cosmic rays transition from being Galactic to becoming extragalactic? And the most basic question remains, 5. what are the sources (and the acceleration mechanism) of ultrahigh energy cosmic rays? To answer these questions more observations are needed. Chief among the theorists' wish list is the increase in statistics at the highest energies and the second wish is for full sky coverage. These efforts should lead to the localization of a source (or sources) in the sky which would revolutionize the field. Another avenue for major progress would be the detection of neutrino and photon secondaries, especially at ultrahigh energies. More immediate progress can be reached with a better establishment of an absolute energy scale which has a direct impact on many models. Another fertile area is the ongoing tests of hadronic interaction models with the LHC. More precise measurements of airshower properties may also enable the distinction between primary composition and hadronic physics effects on a wide energy range. Joint anisotropy and composition analysis between the two leading experiments (Auger and TA) would also provide a much needed consistency between the two hemispheres. This meeting marks the beginning of a great effort in this direction.
\end{abstract}

\section{INTRODUCTION}

The field of ultrahigh energy cosmic rays (UHECRs) has greatly profited from the effort by theorists and phenomenologists to make the best use of the limited data available at the highest energies. For instance, the main question in the field at the turn of the century had been posed by the theoretical realization made in 1966 by Greisen, Zatsepin and Kuzmin (GZK) [1, 2] that the cosmologically produced cosmic microwave background (CMB) would strongly affect the propagation of cosmic rays of ultrahigh energies. The existence of the GZK effect had been already challenged by observations in 1963 of a trans-GZK event (and event above $60 \mathrm{EeV}$ ) by Linsley [3], but much higher statistics observations by AGASA [4] suggested the need to go beyond known physics to explain the absence of a

\footnotetext{
ae-mail: olinto@kicp.uchicago.edu
}

This is an Open Access article distributed under the terms of the Creative Commons Attribution License 2.0, which permits unrestricted use, distribution, and reproduction in any medium, provided the original work is properly cited. 


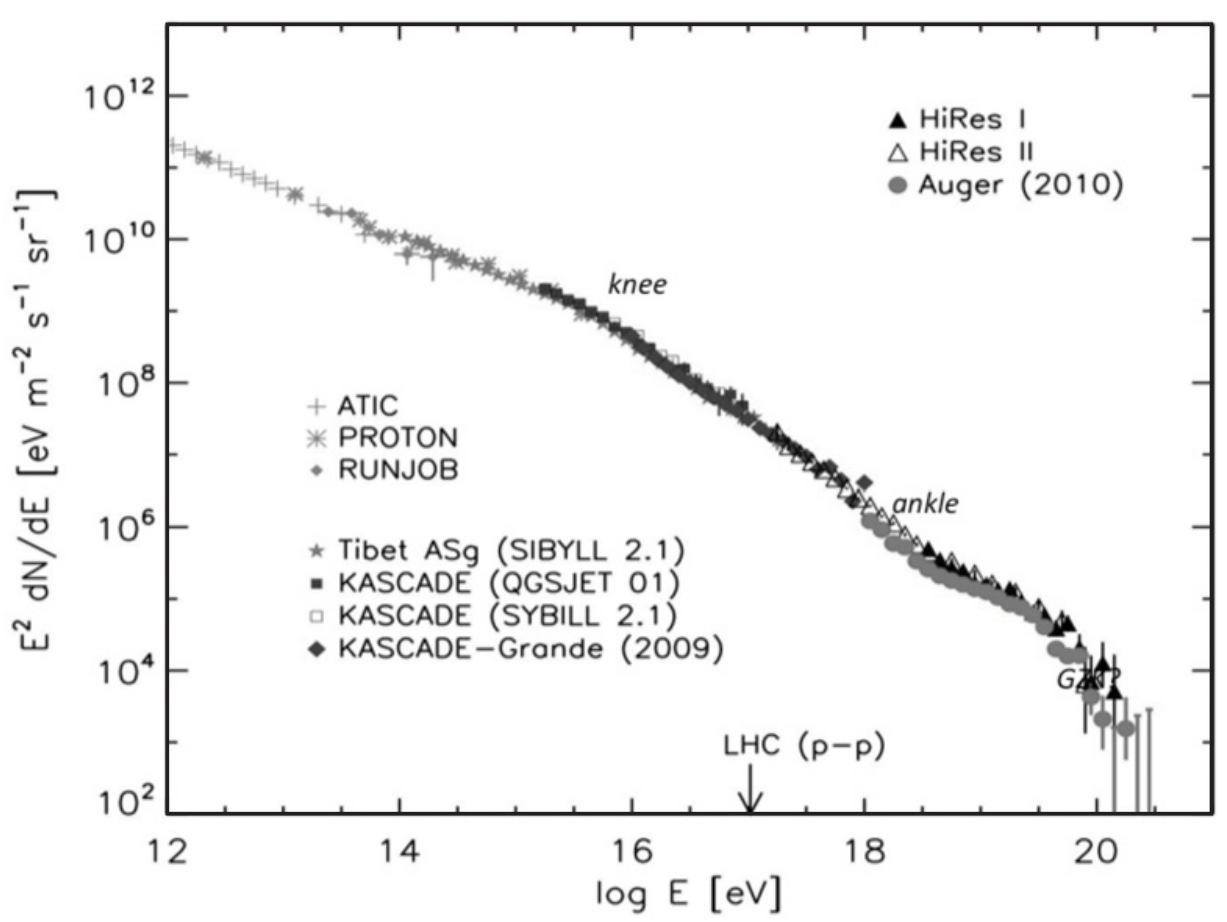

Figure 1. All particle cosmic ray flux multiplied by $E^{2}$ observed by ATIC [13], Proton [14], RUNJOB [15], Tibet AS- $\gamma$ [16], KASCADE [17], KASCADE-Grande [18], HiRes-I [9], HiRes-II [8], and Auger [11]. LHC energy reach of $p-p$ collisions (in the frame of a proton) is indicated for comparison. (Adapted from [19]).

GZK feature. This puzzle inspired the construction of the current giant observatories: the Pierre Auger Observatory in Argentina [5] and the Telescope Array in Utah [6].

The question of the existence of a GZK feature in the spectrum above tens of $\mathrm{EeV}\left(\mathrm{EeV}=10^{18} \mathrm{eV}\right)$ was successfully answered by the most recent generation of UHECR observatories, beginning with the High Resolution Fly's Eye (HiRes) results [7-9] followed by a significant jump in statistics by the Pierre Auger Observatory [10, 11], and most recently the Telescope Array (TA) [12]. Figure 1 shows a compilation of the cosmic ray spectrum (multiplied by $E^{2}$ ) from $\mathrm{TeV}\left(=10^{12} \mathrm{eV}\right)$ to $\mathrm{ZeV}$ $\left(\mathrm{ZeV}=10^{21} \mathrm{eV}\right)$ energies. The knee, between 1 and $10 \mathrm{PeV}\left(\mathrm{PeV}=10^{15} \mathrm{eV}\right)$, and the ankle, between 1 and $10 \mathrm{EeV}$, are indicated as well as the equivalent interaction energy reach of the Large Hadron Collider (LHC) at $100 \mathrm{PeV}$. As displayed in Fig. 2, the flux (multiplied by $E^{3}$ ) of UHECRs shows the clear presence of a GZK-like feature. One important open issue is the value of the absolute energy scale that is appropriate for these data since the two largest experiments differ by $20 \%$ overall energy shift. The shape of the spectrum with an absolute energy scale can help select between different theoretical models. For example, the energy scale adopted by TA gives the best fit for models where the UHECR primaries are all protons and the ankle is explained by a dip due to the production of electrons and positrons [20], while the Auger energy scale is well explained by mixed composition models as in, e.g., $[21,22]$.

The great success of observing what GZK predicted in 1966 has shown that there is no need to go beyond known physics to explain the observed spectrum. In addition to following the predicted GZK shape, many alternative models based on UHECR production due to decay of extremely high energy relics from the Early Universe (jointly named Top-down) have been strongly constrained by the lack of ultrahigh energy photons $[23,24]$. Top-down models may still contribute at some level, but are not the dominant source of UHECRs. 


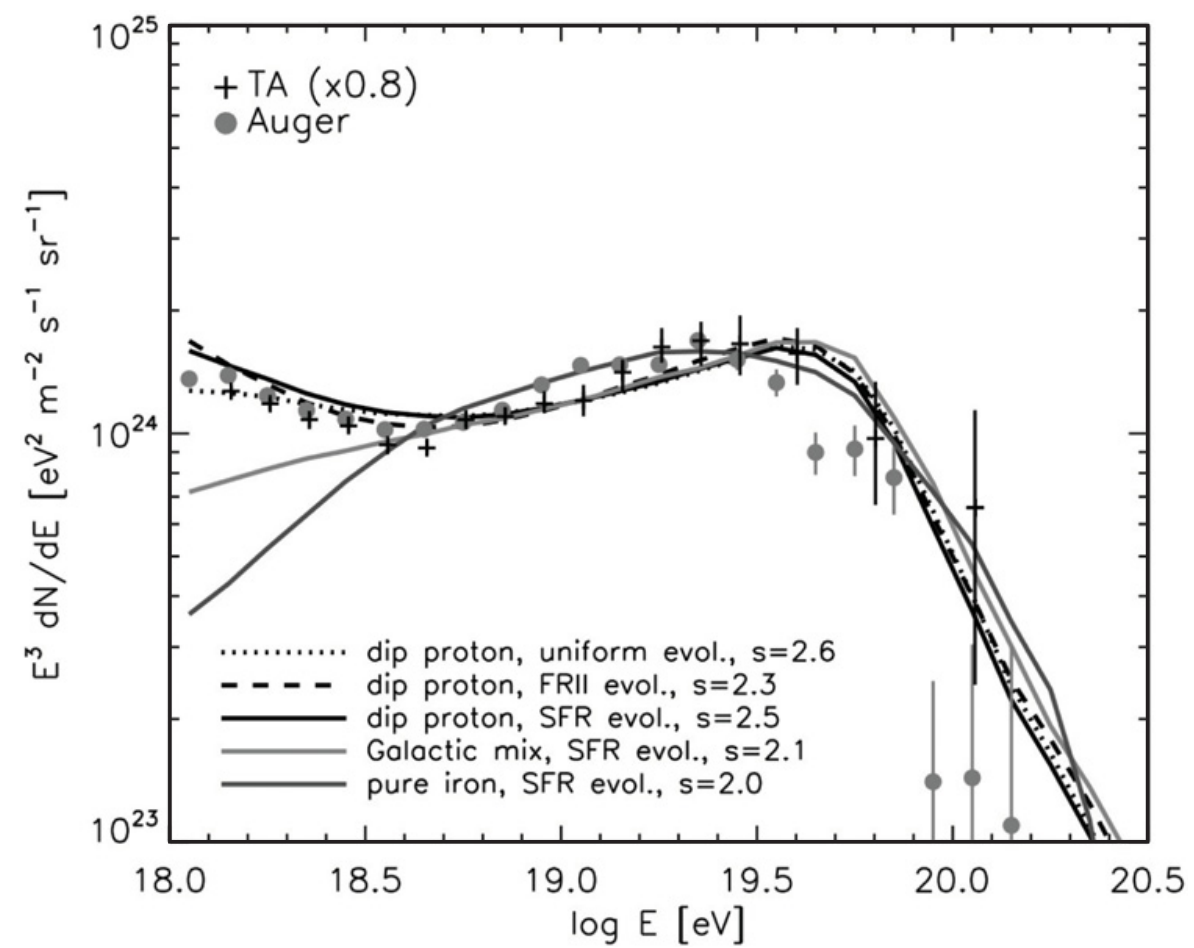

Figure 2. Flux of UHECRs multiplied by $E^{3}$ versus energy from Auger [10] and TA [12] (the TA absolute energy has been multiplied by 0.8). The displayed error bars are statistical errors while the reported systematic error on the absolute energy scale is $22 \%$. Overlaid are simulated spectra obtained for different models of the Galactic to extragalactic transition (ankle or "dip" transition) and different injected chemical compositions (pure protons, Galactic mix, and pure iron) and spectral indices, $s$ from 2 to 2.6 (adapted from [19]).

The observed GZK-like feature supports the well-accepted notion that UHECRs come from powerful extragalactic astrophysical sources distributed throughout the Universe. However, the observed feature does not differentiate between an effect due to the maximum energy of astrophysical accelerators, $E_{\max }$, or the losses due to propagation through cosmological distances. For instance, mixed composition models that explain the observed composition trend seen by Auger [25] rely on the fact that astrophysical accelerators may reach maximum energy around $E_{\max }(Z) \lesssim Z 10 \mathrm{EeV}$, such that the change in composition is due to the change in the dominant primary at a given energy starting with protons around a few EeV and ending with a Carbon or Iron peak just below $100 \mathrm{EeV}$. Figure 3 shows the well-known Hillas plot [26] where astrophysical accelerators are shown with their magnetic fields, $B$, versus their typical sizes $R$. The condition of containment within the Larmor radius, $r_{\mathrm{L}}=E / Z e B \sim 110 \mathrm{kpc}^{-1}(\mu G / B)(E / 100 \mathrm{EeV})$, sets the scale for $E_{\max } \sim 1 \mathrm{EeVZ}(B / 1 \mu \mathrm{G})(R / 1 \mathrm{kpc})$. Efficiencies and corrections due to relativistic effects decrease the actual reach of an accelerator bringing down $E_{\max }$ further. It is clear from the figure that reaching $100 \mathrm{EeV}$ with protons is not trivial. Mixed composition models relax the challenges of the acceleration to such high energies by increasing the particle charge, however most proposed astrophysical acceleration sites are highly proton dominated environments, except for the birth of compact objects in massive stelar progenitors or possibly the environment close to supermassive black holes. The observation of heavier elements at the highest energies narrows down significantly the possible acceleration sites.

A precise measurement of the spectrum above $100 \mathrm{EeV}$ may be able to rule out the $E_{\max }$ explanation of the GZK-like feature, if a recovery is observed as shown in Fig. 4. Plotted are modification 


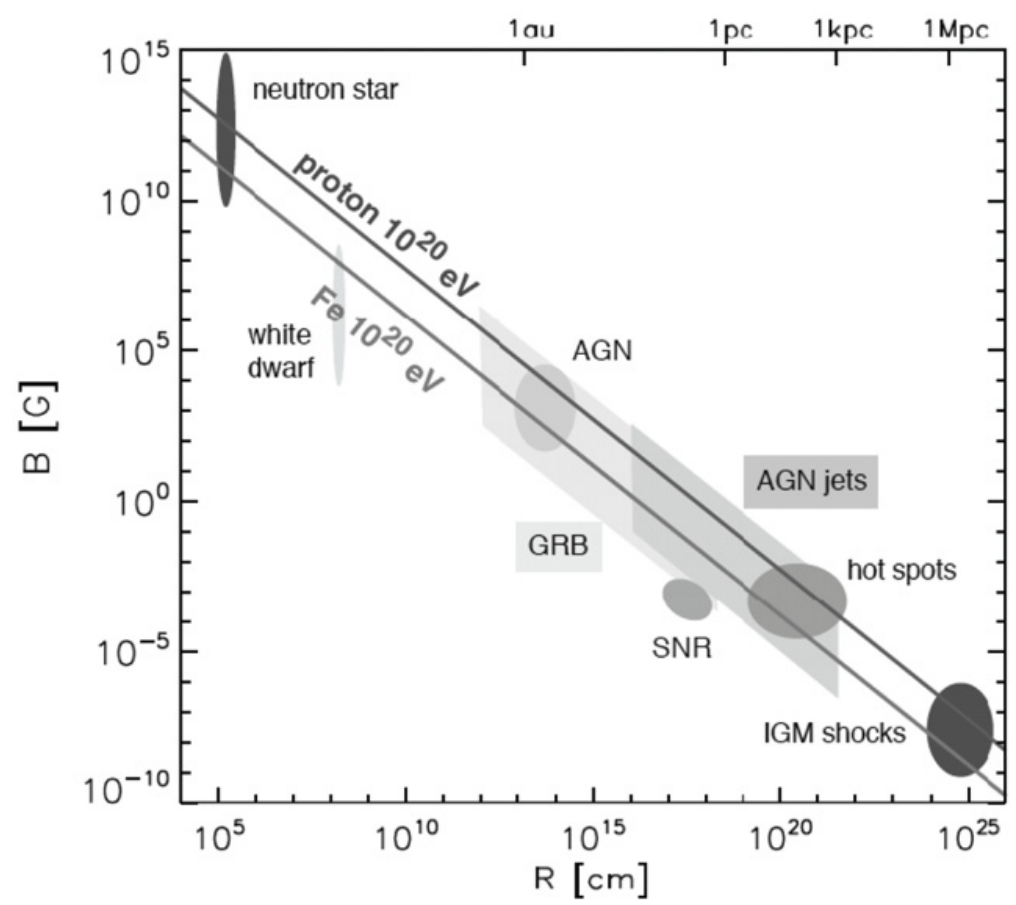

Figure 3. Hillas diagram of magnetic fields versus size of candidate UHECR sources. Above the diagonal lines protons or iron nuclei (as labelled) can be confined to a maximum energy of $E_{\max }=10^{20} \mathrm{eV}$. The most powerful candidate sources are shown with the uncertainties in their parameters. (Adapted from [19]).
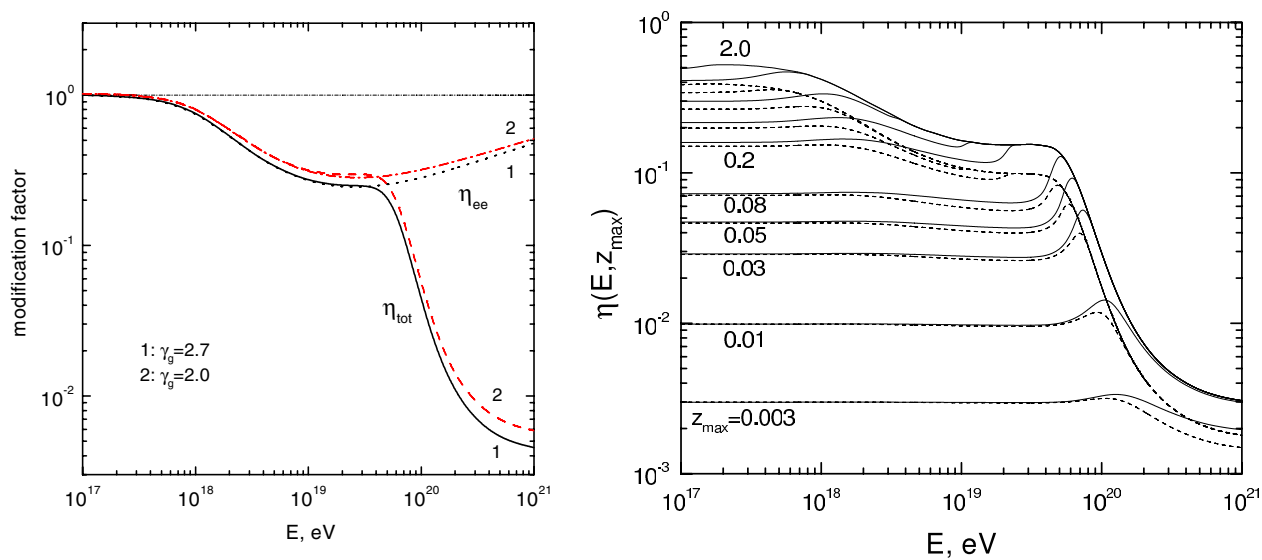

Figure 4. Modification factors for power-law source spectra with slopes 2.0 and 2.7. Left: horizontal line for adiabatic losses, $\eta_{e e}$ for pair production, and $\eta_{t o t}$ for total losses. Right: $\eta$ as a function of redshift for uniform source distribution up to maximum redshift $z_{\max }$. (Adapted from $[27,28]$.)

factors $\eta$ defined as the ratio of the spectrum with all energy losses taken into account (which would be observed on Earth) to the unmodified source spectrum where only adiabatic energy losses (red shift) are included [27, 28]. The figure shows that above $100 \mathrm{EeV}$ the spectrum can have a recovery if sources have $E_{\max } \gg E_{\mathrm{GZK}}$ and are present within redshifts below 0.01 for the powerlaw parameters chosen in this study. However the observational challenge of reaching large number 


\section{UHECR 2012}

of events above $100 \mathrm{EeV}$ awaits a strong international effort or a breakthrough in detection techniques or both. A large increase in statistics at these highest energies will also enable the observation of anisotropies in the sky distribution which could signal the first direct detection of UHECR sources.

Below I briefly summarize the theoretical and phenomenological contributions to the UHECR2012 Symposium. It is clear that great progress occurred in modeling hadronic interactions which enabled very successful tests of the models at the Large Hadron Collider (LHC) and a better control of the extrapolations to the untested territory of $\gtrsim 10 \mathrm{TeV}$ center-of-mass (CM) energies (see, e.g., $[29,30])$. Cosmic ray propagation models have been developed for ultrahigh energy protons, nuclei, photons, and neutrinos which include all the interactions with known photon background fields, from the cosmic microwave background (CMB) to the infrared, optical, and ultraviolet radiation fields (IR-UV) (see, e.g., [31-35]). The "known unknown" structure of the cosmic magnetic fields permeating the extragalactic space has been modeled within known constraints (see, e.g., [33, 34]). The observations of the Galactic magnetic field has improved to the level that one can be certain that for energies above $20 \mathrm{EeV}$ cosmic rays are extragalactic (unless they have higher charges than Iron) [36, 37]. This great result makes use of the observed constraints on the dipole anisotropy up to these energies. Propagation codes such as CRPropa [38] and SimProp [39] have been made available to the community so different ideas can be easily translated from source models to observed spectrum, composition, and sky distribution on Earth [32-34]. Source models have been developed for all known photon accelerators, from the birth of compact objects such as black holes and neutron stars (gamma-ray bursts, young pulsars, magnetars) to larger structures such as active galactic nuclei (AGN) and their radio lobes up to supercluster shocks (see, e.g., [32, 35, 40, 41]). Multimessenger approaches to unveiling the origin of UHECRs have made great use of recent gamma-ray observations and neutrino limits [32, 35, 42, 43]. Finally, an interesting new physics proposal based on chiral symmetry restoration [44] was designed to reconcile the shower properties observed by Auger [25] with a proton primary composition. Clearly theorists are well deserving of a good bottle of wine.

\section{SUMMARY}

Observations of UHECRs should allow a study of hadronic interactions at energies well above particle accelerators. However, the ambiguity between measuring the composition of UHECR primaries and studying the hadronic interaction complicates this task. This challenge was highlighted by [30], who proposed to separate hadronic interactions from composition effects by extending the method based on the shape of the $X_{\max }$ distribution that allows a measurement of the proton-air interaction length, pioneered by Fly's Eye and recently applied by Auger to constrain the proton-air cross section at CM energy per nucleon of $57 \mathrm{TeV}$ [45]. This type of study extended through different energy bins can constrain the energy dependence of the proton-air interaction length, the evolution of the chemical composition, and some global properties of hadronic interactions at these extreme energies.

Recent progress in modeling hadronic interactions was shown by [29] who discussed the connection between accelerator experiments and parameters relevant for cosmic ray showers. In particular, the inelastic cross sections, the secondary particle multiplicity, and the inelasticity influence predictions for the mean depth of shower maximum, $\left\langle X_{\max }\right\rangle$. With LHC data at $7 \mathrm{TeV}$, the uncertainty in extrapolating to the highest energies has decreased by a factor of $\sim 2$ in $\left\langle X_{\max }\right\rangle$ when comparing QGSJETII and EPOS models, which now have differences of $\sim 20 \mathrm{~g} / \mathrm{cm}^{2}$ which is comparable to the experimental uncertainties (QGSJETII-04 now gives larger $\left\langle X_{\max }\right\rangle$ which implies heavier primaries in the Auger data). The tests by LHC data on mid-rapidity (anti)baryon and strangeness production and low energy fixed-target pion production have brought QGSJETII closer to the EPOS model prediction for the muon numbers, making the problem of too many muons in UHECR data not as extreme. 
The large number of muons in Auger observations as compared to hadronic model predictions [46] and the surprising trend of $\left\langle X_{\max }\right\rangle$ and RMS ( $X_{\max }$ [25] above $10 \mathrm{EeV}$ may be explained by an important change in particle physics at these energies. In this meeting, [44] presented a model that clarifies the changes needed to explain the trends seen in the longitudinal and lateral shower development properties by Auger. The model is based on chiral symmetry restoration that can lead to the suppression of $\pi^{0}$ decays when compared to current models. The decrease in the spread of current hadronic model predictions [29] is also encouraging, as the need for a significant change can only be clarified if the extrapolation of known physics has a narrow range of extrapolations.

The transition between Galactic and extragalactic cosmic rays has to occur at energies below $20 \mathrm{EeV}$ according to studies of [36]. This conclusion is based on the anisotropy that nuclei up from $Z=1$ to 26 would induce in the sky distribution of UHECRs given a Galactic origin, the known properties of the Galactic magnetic field, and the Auger limits on the dipole up to $20 \mathrm{EeV}$ [47]. In addition, they conclude that if protons dominate the cosmic ray component between 0.1 and $1 \mathrm{EeV}$, they should be of extragalactic origin, while higher charges can still be acommodated as Galactic up to the ankle or beyond (in the case of Iron). They also reported on the (de-)magnification of the flux and image of UHE nuclei sources due to the structure of the Galactic magnetic field [37].

The relationship between the shape of the spectrum, the lack of strong anisotropies, and the composition of cosmic rays between $10 \mathrm{PeV}$ and $10 \mathrm{EeV}$ can strongly constrain different models for the origin of UHECRs as highlighted by $[31,32,34]$. As these studies show, it is very important for observations to reach a clearer picture of both the shape and energy scale of the UHECR spectrum and the composition trends from $10 \mathrm{PeV}$ to $100 \mathrm{EeV}$. Dip models that fit the ankle with pair production losses have a fixed energy scale for the ankle, can explain the possible presence of a second knee, and are dominated by protons from smaller energies. In contrast, models with mixed compositions argue for a transition at the ankle, a heavier composition up to ankle energies, a return to light compositions at the ankle followed by heavier composition as energies reach $E_{\max }$. scales. Both dip and ankle models have many parameters such as the injection spectrum and composition and the cosmological evolution, therefore, more guidance from observations are needed to reach a firm conclusion. For instance, the Auger composition argues for sources of nearby UHE heavy nuclei with a hard injection spectrum [35], such as the pulsar model I describe below. But the HiRes and TA composition analyses do not corroborate the Auger $X_{\max }$ results thus far. (At these energies a change in composition as a function of the Earth's hemisphere seems too senseless, even for the most irreverent theorists, so the problem should be an experimental one.) The connection with lower energy cosmic rays is also important as acceleration at supernovae shocks have been shown to fit well the spectrum at lower energies up to a $10 \mathrm{PeV}$ [32] but not up to ankle energies. The computational tools available for extragalactic propagation studies have greatly improved and were discussed by $[33,34,37]$.

A great resource for placing constraints on proposed UHECR sources is multi-messenger information provided by photons (from radio to gamma-rays) and eventually by neutrinos and maybe even ultrahigh energy photons. Clearly the large knowledge from photon observations is the basis for all astrophysical models of UHECR sources. Of these classes of models, transients large luminosity events are likely sites of acceleration which can explain the difficulty in finding strong anisotropies for larger source densities. A strategy for unveiling transient sources was presented by [40] where source densities and transient time profiles are used to constrain the source parameters. The distribution of source properties, such as black-hole masses for AGN models, also affects predictions of UHECR observables as discussed by [41]. As discussed by [42], recent gamma-ray observations of blazars may require the acceleration of UHECRs in AGNs. Future gamma-ray observations may confirm this interesting possibility.

High energy neutrinos are the messengers most intimately connected with hadronic acceleration processes and therefore with UHECR sources and propagation. As discussed by [33], the observations of neutrinos from $\mathrm{PeV}$ to $\mathrm{ZeV}$ can strongly constrain models for the origin of UHECRs. Figure 5 shows the predictions from a range of models of the neutrinos produced by the propagation of UHECRs and the 


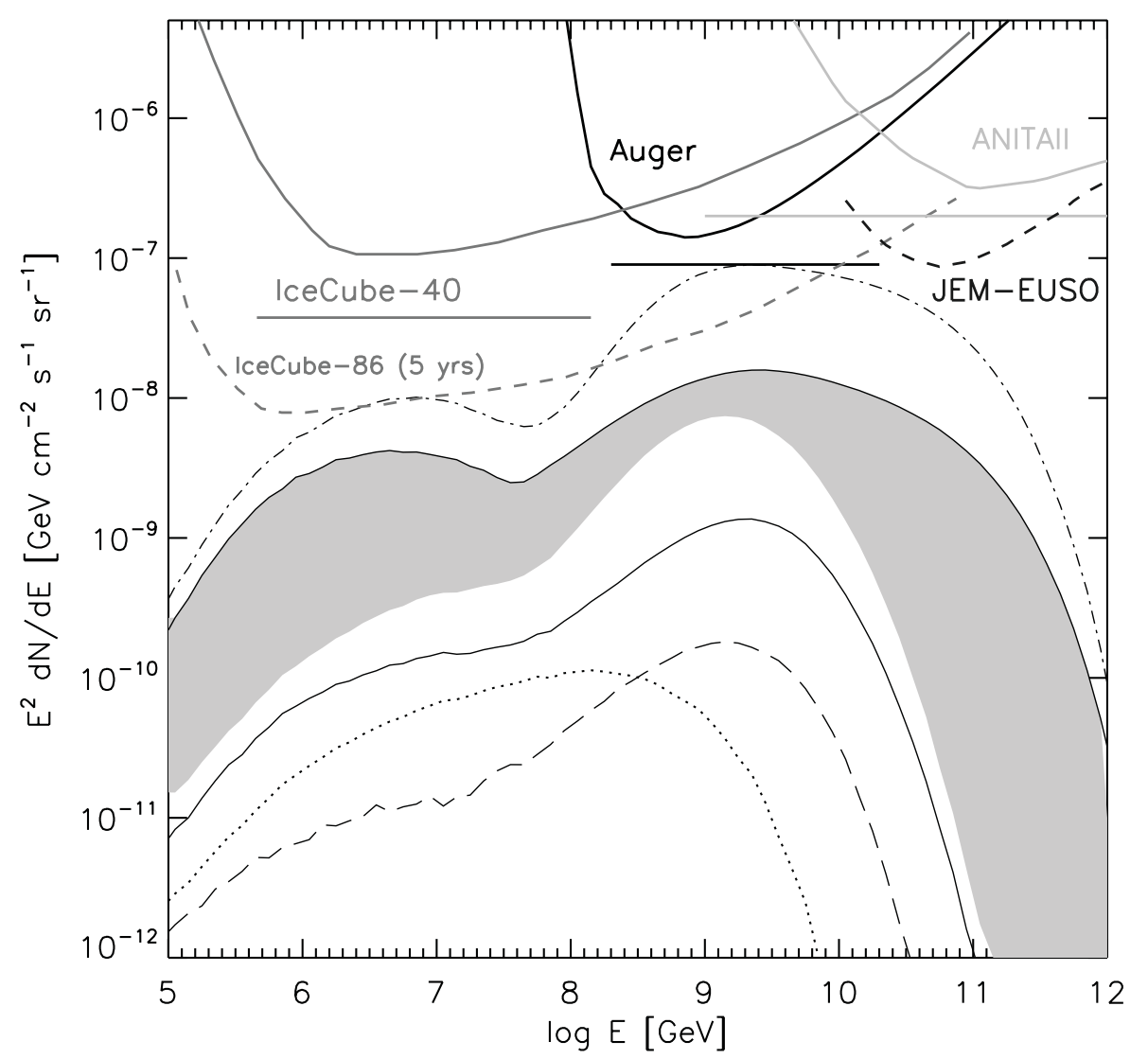

Figure 5. Cosmogenic neutrino flux for all flavors, for different UHECR parameters compared to instrument sensitivities (adapted from [48]). Dash-dotted line corresponds to a strong source evolution case (FRII evolution, see [49]) with a pure proton composition, dip transition model, and $E_{\max }=3 \mathrm{ZeV}$. Uniform source evolution with: iron rich (30\%) composition and $E_{Z, \max }<Z 10 \mathrm{EeV}$ is shown in the dotted line and the dashed line is for pure iron injection and $E_{Z, \max }=Z 100 \mathrm{EeV}$. Grey shaded range brackets dip and ankle transition models, with evolution of star formation history for $z<4$, pure proton and mixed 'Galactic' compositions, and large proton $\left.E_{\max }(>100 \mathrm{EeV})\right)$. Including the uniform source evolution would broaden the shaded area down to the black solid line. Experimental limits (solid lines) assume 90\% confidence level and full mixing neutrino oscillation. The differential limit and the integral flux limit on a pure $E^{-2}$ spectrum (straight line) are presented for IceCube-22 [50], Ice-Cube-40 [51], ANITA-II [52] and Auger [53]. Dashed lines show future sensitivities for IceCube 80 lines [54], and for JEM-EUSO [55]).

reach of current experiments. The 3 order of magnitude range is an experimental challenge, but recent reports from IceCube of two $\mathrm{PeV}$ cascade events may be the beginning of a new era.

\section{OUTLOOK}

Over the last decade, UHECR observations have settled a number of open questions such as the existence of a spectral feature above $10 \mathrm{EeV}$ consistent with the Greisen-Zatsepin-Kuzmin (GZK) prediction, the clear presence of the ankle, the lack of more AGASA doublets [56], hints of anisotropies on large angular scales for trans-GZK events (above $60 \mathrm{EeV}$ ), and an interesting trend in composition or hadronic interactions above $10 \mathrm{EeV}$. LHC data has also helped sharpen hadronic interaction models and decrease the energy gap for extrapolations (see, e.g., [29]). However, many questions remain. As theoretical 


\section{EPJ Web of Conferences}

models of UHECRs adapt to better measured spectra and the lack of strong anisotropies, current open questions can be framed as:

1. Is the spectral feature at the highest energies the GZK cutoff or the effect of $E_{\max }$ ?

2. Is the composition of primaries changing above $10 \mathrm{EeV}$ or are new interactions responsible for the change in behavior of extensive air showers?

3. At what energy and sensitivity will sources be observed in the sky distribution?

4. At what energies cosmic rays transition from being Galactic to becoming extragalactic? And the most basic question,

5. what are the sources (and the acceleration mechanism) that produce ultrahigh energy cosmic rays?

These questions are clearly related to each other and a given model should have answers to all. For example, if we take the class of models that assumes a "dip transition"[20], then the answer to 4 is at energies well bellow the ankle $(\sim 0.1 \mathrm{EeV}$, e.g., $[32,34])$. Then the composition has to be proton dominated [21] and the answer to 2 is then new hadronic interactions, e.g., [44]. The answer to 1 is most likely a GZK feature, as there is no need in such models to assume $E_{\max }$ to be close to $E_{\mathrm{GZK}}$. One difficulty for these models is question 3, as protons should start pointing at observed energies (see, e.g., [57]), but transient sources and stronger extragalactic magnetic fields may delay significant anisotropies to higher energies. Within such models there are still many possibilities for answers to question 5 as shock acceleration is a reasonable mechanism that occurs in gamma-ray bursts, AGNs, and possibly other sites. Some predictions of this class of models would be a well defined energy for the ankle, since pair production has a fixed energy scale (i.e., the HiRes energy scale is the correct one), clustering of events at higher energies, a proton dominated composition, and possibly a recovery of the spectrum at higher energies (if $E_{\max } \gg E_{\mathrm{GZK}}$ ). If the proton composition can be determined astrophysically, for example, through source images, then changes in hadronic interactions should explain the Auger behavior of $X_{\max }$. Testing this model would involve accumulating large statistics above $60 \mathrm{EeV}$ to observe both the spectrum, the anisotropies, and possibly to test hadronic interactions. The determination of an absolute energy scale and the separation between primary composition and hadronic interactions in the observations of shower development would also be of great help. Secondary neutrinos are more abundant then in mixed composition models and may be within reach of current or future detectors.

If instead we chose to answer question 2 with a change in composition to fit the Auger observations of $\left\langle X_{\max }\right\rangle$ and $\operatorname{RMS}\left(X_{\max }\right)$ as shown, for example in Fig. 6, then the injection spectrum needs to be harder then in most models, i.e., $\propto E^{-\alpha}$ and $\alpha<2$ [35]. This kind of hard spectrum in difficult for shock acceleration based models, so alternative mechanisms have to be at play. A reasonable alternative is the hard spectrum expected in young pulsar acceleration models. The pulsar spins down due to magnetic breaking naturally giving rise to a $\alpha=1$ spectrum [66]. The escape from the young supernova remnant (SNR) softens the spectrum to $\alpha \simeq 1.4$ [65]. In addition, the surface of the pulsar is Iron rich and the progenitor is Oxygen rich, so the site is a natural for acceleration of heavier nuclei, with the answer to question 3 being primaries have larger $Z$ which delay anisotropy signals in energy by $Z$ with respect to protons. In this model, $E_{\max }$ (proton) $\ll E_{\mathrm{GZK}}$ as the maximum spin rate of neutron stars is fixed by the neutron star structure, then answer to 1 is the effect of $E_{\max }(Z=26)$. The answer to 4 is the transition from Galactic to extragalactic happens at the ankle, but the role of Galactic pulsars may dominate the cosmic ray spectrum below the ankle if the absolute energy scale is that of HiRes [22] as shown in Fig. 7. Finally the answer to 5 is more specific to young pulsars where the $E_{\max }$ is determined by the physics of neutron stars and the acceleration is related to the pulsar spin down rate. There are other sites which may have higher abundances of heavier elements or a natural limit on $E_{\max }$. Testing this type of model will again require large statistics above $60 \mathrm{EeV}$ to observe anisotropies and the shape of the spectrum around $100 \mathrm{EeV}$. The absolute energy scale would determine the dominance of Galactic pulsars in Galactic cosmic rays. A separation between primary composition and hadronic interactions 

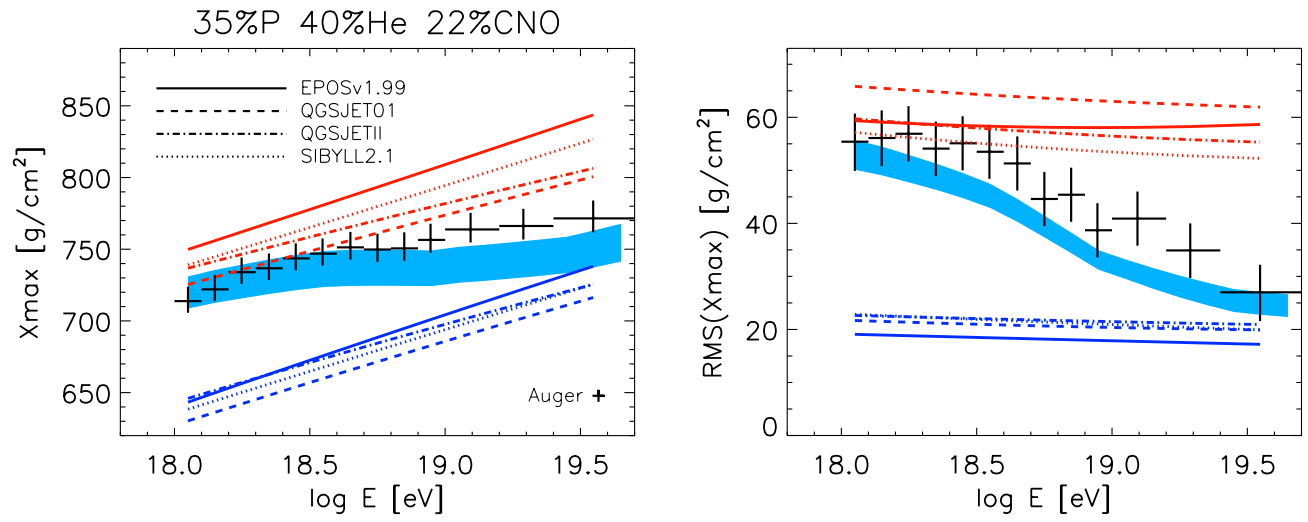

Figure 6. $\left\langle X_{\max }\right\rangle$ (top panel) and $\mathrm{RMS}\left(X_{\max }\right)$ (bottom panel) of Auger data (crosses) [25] and results of the pulsar model of [22] (bars) with mixed composition of 35\% Proton, 40\% Helium, 22\% CNO and 3\% Fe nuclei. The range of predictions are due to the four hadronic interaction models considered as listed in the legend. (Adapted from [22].)
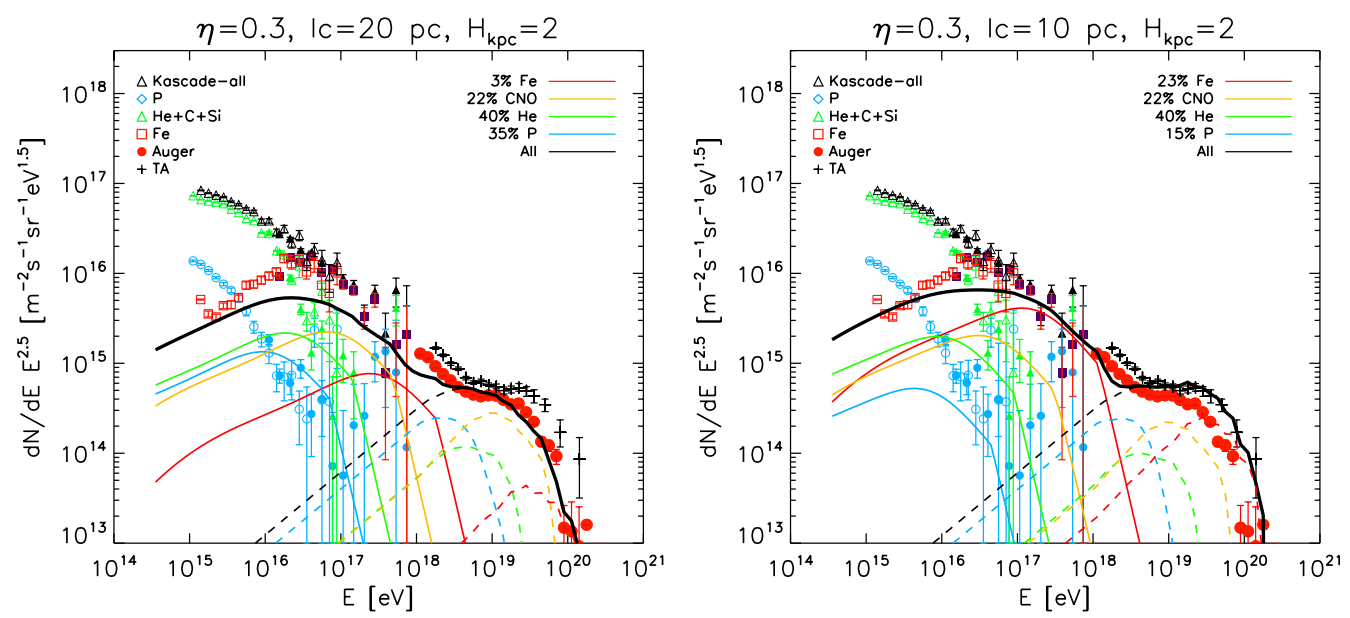

Figure 7. Cosmic ray flux measurements by KASCADE-Grande [18], Auger [10], and TA [12] compared with pulsar model predictions of [22]. The total spectrum in solid black sums up extragalactic (dash) and Galactic (solid) components. Top panel the Auger case: 35\% Proton, 40\% Helium, 22\% CNO and 3\% Fe. Bottom panel: the TA case of $15 \%$ Proton, $40 \%$ Helium, $22 \% \mathrm{CNO}$ and $23 \% \mathrm{Fe}$. Pulsar and propagation parameters: wind acceleration coefficient $\eta=0.3$, Galactic magnetic field coherence length $l_{c}=10 \mathrm{pc}$, magnetic halo height $H=2 \mathrm{kpc}$. (Adapted from [22].)

in the observations of shower development would also be of great help. Another interesting prediction is that the flux of cosmic rays between 0.1 and $1 \mathrm{EeV}$ can be highly dependent on the supernova history of the Galaxy and would become stronger (and anisotropic) should a nearby young pulsar be born.

Clearly theorists have tried a number of models that can explain a given aspect of current data, but answering these questions will take a consorted effort by the whole community. Theorists and phenomenologists can improve on the model predictions and test them not only with UHECR data but also with a large number of multi-messenger information being collected with photons (from radio to gamma-rays) and neutrinos (e.g., two cosmic neutrinos of $\mathrm{PeV}$ energies were recently reported by IceCube [58]). The particle physics effort will certainly gain with LHC data and deeper understanding 


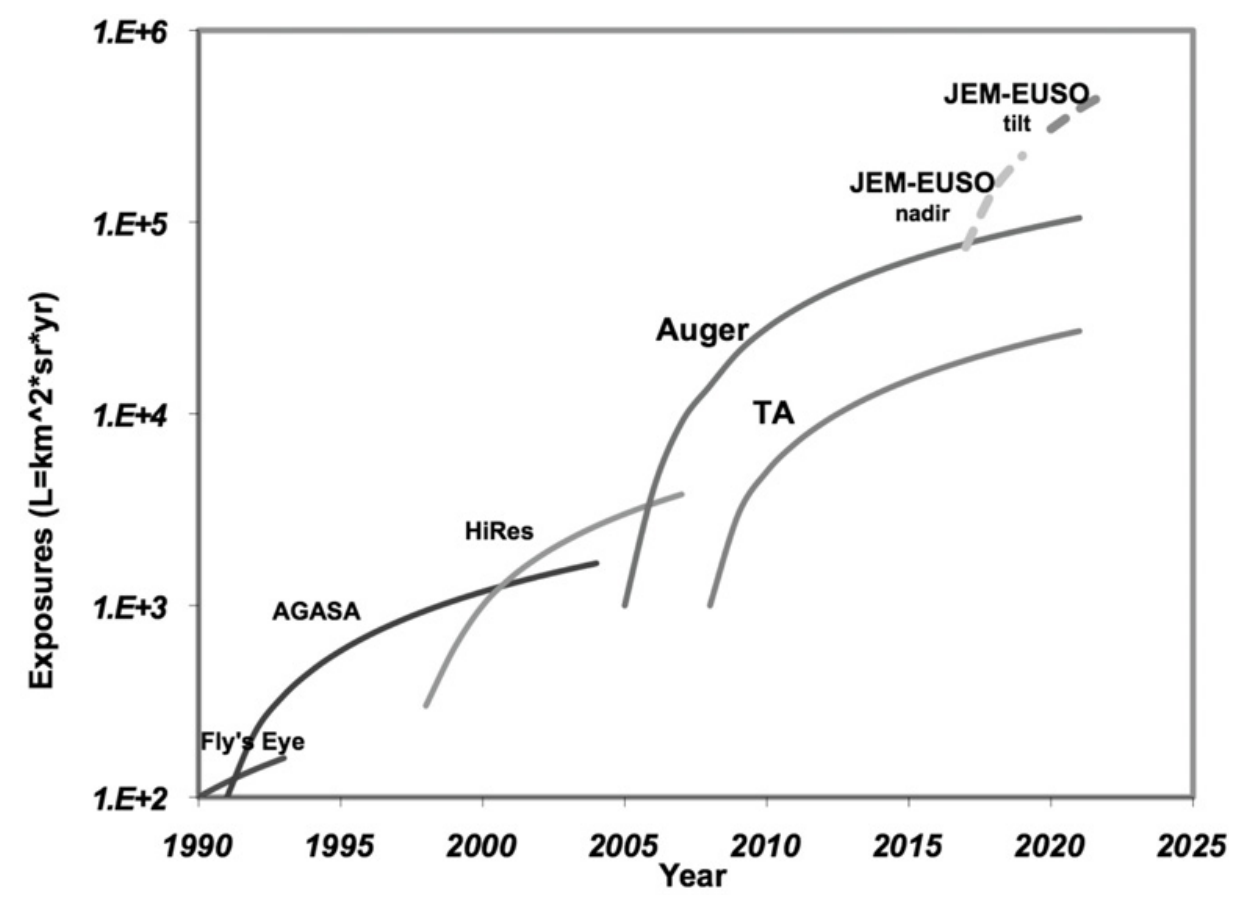

Figure 8. Exposures to UHECRs from 1990 to present from Fly's Eye, AGASA, HiRes, Auger and TA. Estimates of the exposures in the future of Auger and TA plus the planned space observatory, JEM-EUSO [55].

of the observed shower properties. However, the observational challenge at ultrahigh energies is crucial to solving this puzzle. Top on many theorists wish list is the increase of statistics above $60 \mathrm{EeV}$.

A picture with $10^{3}$ trans-GZK events is worth $10^{3}$ times a thousand words. Why $10^{3}$ trans-GZK events? The indication that above $60 \mathrm{EeV}$ the distributions of arrival directions is better matched to anisotropic distributions such as the nearby AGN distribution [59] or the clustering of large scale structures $[60,61]$ than to an isotropic distribution indicates that increasing the statistics above $60 \mathrm{EeV}$ will sharpen the image which should be even clearer at $100 \mathrm{EeV}$. A reasonable estimate of the number of events that would be needed can be attained via different arguments, but the number of $10^{3}$ comes out of at least two studies: the number of events needed to reach a $5 \sigma$ discovery of a dipole towards Cen A (with the amplitude of 0.25 as presently indicated by Auger) is $10^{3}[62,63]$ which is also the estimated number of events to separate different populations of sources via their distribution in the magnetized large scale structure [64].

Present experiments accumulate less than 30 trans-GZK events per year while the Earth receives a few $10^{6}$ events per year. As shown in 8 , the field has progressed steadily over the last two decades. To continue the effort to bridge these 5 orders of magnitude, the JEM-EUSO collaboration proposes to install a fluorescence telescope on the International Space Station by 2017 [55]. This step would open the $10^{3}$ trans-GZK events window and begin the systematic observations of these puzzling particles from space.

Thanks to the organizers for an excellent Symposium. Thanks also to K. Fang, K. Kotera, P. Blasi, and colleagues in Auger and JEM-EUSO for very fruitful discussions. This work was supported by the NSF grant PHY-1068696 at the University of Chicago, and the Kavli Institute for Cosmological Physics through grant NSF PHY-1125897 and an endowment from the Kavli Foundation. 


\section{UHECR 2012}

\section{References}

[1] K. Greisen, Phys. Rev. Lett. 16 (1966) 748

[2] G.T. Zatsepin and V.A. Kuz'min, JETP Lett. 4 (1966) 78

[3] J. Linsley, Phys. Rev. Lett. 10 (1963) 146

[4] M. Takeda et al., Phys. Rev. Lett. 81 (1998) 1163

[5] J. Abraham et al., Nucl. Instrum. Meth. in Phys. Res. A 523 (2004) 50

[6] T. Abu-Zayyad et al., Nucl. Instrum. Meth. in Phys. Res. A 689 (2012) 87

[7] R.U. Abbasi et al., Phys. Rev. Lett. 92 (2004) 1101

[8] R. U. Abbasi, et al. (HiRes Collab), Phys. Rev. Lett. 100 (2008) 10

[9] R. U. Abbasi, et al., Astroparticle Physics 32 (2009) 53

[10] J. Abraham, et al., Phys. Rev. Lett. 101 (2008) 1101

[11] J. Abraham, et al., Phys. Lett. B 685 (2010) 239

[12] T. Abu-Zayyad et al., preprint arXiv: 1205.5067

[13] H. S. Ahn, et al., (ATIC Collab) International Cosmic Ray Conference 2 (2008) 79

[14] N. L. Grigorov, et al. (PROTON Collab), International Cosmic Ray Conference 1 (1971) 172

[15] A. V Apanasenko, et al. (RUNJOB Collab), International Cosmic Ray Conference 5 (2001) 1622

[16] D. Cheng et al. (Tibet AS- $\gamma$ ), International Cosmic Ray Conference 4 (2008) 103

[17] K.-H. Kampert, et al. (KASCADE Collab), Nuclear Physics B Proc. Suppl. 136 (2004) 273

[18] W. D. Apel, et al., Proc. 32nd ICRC (2011) arXiv:1111.5436

[19] K. Kotera, A. V. Olinto, Annu. Rev. Astron. Astrophys. 49 (2011) 119

[20] V. Berezinsky, A.Z. Gazizov and S.I. Grigorieva, Phys. Lett. B 612 (2005) 147

[21] D. Allard, E. Parizot, A.V. Olinto, E. Khan and S. Goriely, A\&A 443 (2005) L29

[22] K. Fang, K. Kotera, A.V. Olinto, JCAP, submitted (2012)

[23] J. Abraham, et al., Astropart. Phys. 31 (2009) 399

[24] J. Abraham, et al., Astropart. Phys. 2008, 29243

[25] J. Abraham, et al., Phys. Rev. Lett. 104 (2010) 091101

[26] A. M. Hillas, ARAA 22 (1984) 425

[27] V.S. Berezinskii and S.I. Grigor'eva, A\&A 199 (1988) 1

[28] 24 R. Aloisio, V. Berezinsky, P. Blasi, A. Gazizov, S. Grigorieva and B. Hnatyk, Astropart. Phys. 27 (2007) 76

[29] T. Pierog, in these proceedings of the UHECR2012 Symposium (2012)

[30] P. Lipari, in these proceedings of the UHECR2012 Symposium (2012)

[31] V. Berezinsky, in these proceedings of the UHECR2012 Symposium (2012)

[32] P. Blasi, in these proceedings of the UHECR2012 Symposium (2012)

[33] G. Sigl, in these proceedings of the UHECR2012 Symposium (2012)

[34] R. Aloisio, in these proceedings of the UHECR2012 Symposium (2012)

[35] A. Taylor, in these proceedings of the UHECR2012 Symposium (2012)

[36] G. Giacinti, M. Kachelriess, D. V. Semikoz, and G. Sigl in these proceedings of the UHECR2012 Symposium (2012)

[37] D. Semikoz, in these proceedings of the UHECR2012 Symposium (2012)

[38] K.-H. Kampert et al., arXiv:1206.3132

[39] R. Aloisio D. Boncioli, A.F. Grillo, S. Petrera, and F. Salamida, arXiv:1204.2970

[40] H. Takami, in these proceedings of the UHECR2012 Symposium (2012)

[41] S. Troitsky, in these proceedings of the UHECR2012 Symposium (2012)

[42] A. Kusenko, in these proceedings of the UHECR2012 Symposium (2012)

[43] D. Fargion, in these proceedings of the UHECR2012 Symposium (2012)

[44] J. Allen and G. Farrar, in these proceedings of the UHECR2012 Symposium (2012)

[45] P. Abreu et al., Phys. Rev. Lett. 109 (2012) 062002

[46] J. Allen for the Pierre Auger Collaboration, Proc. 32nd ICRC, Beijing, China, (2011) 
[47] P. Abreu et al., Astropart. Phys. 34 (2011) 627

[48] K. Kotera, D. Allard, A. V. Olinto, JCAP 10 (2010) 13

[49] J. V. Wall, C. A. Jackson, P. A. Shaver, I. M. Hook, K. I. Kellermann, A\&A 434 (2005) 133

[50] R. U. Abbasi, et al., Phys. Rev. Lett. 104161101

[51] R. U. Abbasi, et al., Phys. Rev. D 84 (2011) 082001

[52] P. W. Gorham, et al., arXiv:1003.2961

[53] J. Abraham, et al., Phys. Rev. D 79 (2009) 102001

[54] A. Karle, IceCube, arXiv: 1003.5715

[55] Y. Takahashi, et al., The Jem-Euso Mission, New J. Phys. 11 (2009) 065009; J. H. Adams Jr et al., 32nd International Cosmic Ray Conference, arXiv:1204.5065

[56] Takeda M et al ., Astrophys. J. 522 (1999) 225; Uchihori Y et al., Astropart. Phys. 13 (2000) 151; Hayashida N et al., astro-ph/0008102

[57] D. De Marco, P. Blasi and A.V. Olinto, JCAP 0607 (2006) 015

[58] A. Ishihara, plenary talk at NU2012, Kyoto

[59] J. Abraham et al., Science 318 (2007) 938; ibid, Astroparticle Physics 29 (2008) 188

[60] P. Abreu et al., Astroparticle Physics 34 (2010) 314

[61] T. Abu-Zayyad et al., Astrophys. J. 757 (2012) 26

[62] L. A. Anchordoqui, H. Goldberg and T. J. Weiler, Phys. Rev. D 84 (2011) 067301

[63] J. H. Adams et al., arXiv:1203.345 (2012)

[64] S. Kalli, M. Lemoine, K. Kotera, A.\& A. 528A (2010) 109

[65] K. Fang, K. Kotera and A.V. Olinto, Ap. J. 750 (2012) 118

[66] P. Blasi, R. Epstein, and A.V. Olinto, Astrophys. J. Lett. 533 (2000) L123 